\title{
Intra-operative motor function preservation for resection of primary motor cortex meningioma
}

\author{
Hailiang Tang ${ }^{1 \#}$, Feng Xu ${ }^{1 \#}$, Liqing Lin ${ }^{2 \#}$, Hongda Zhu ${ }^{1}$, Lingyang Hua ${ }^{1}$, Shihai Luan ${ }^{1}$, Qing Xie ${ }^{1}$, Ye Gong ${ }^{1}$ \\ ${ }^{1}$ Department of Neurosurgery, Huashan Hospital, Fudan University, Shanghai 200040, China; ${ }^{2}$ Department of Neurosurgery, The Affiliated Suzhou \\ Hospital of Nanjing Medical University, Suzhou 215008, China \\ Contributions: (I) Conception and design: H Tang; (II) Administrative support: Q Xie, Y Gong; (III) Provision of study materials or patients: H \\ Zhu, L Hua, S Luan; (IV) Collection and assembly of data: L Lin; (V) Data analysis and interpretation: F Xu; (VI) Manuscript writing: All authors; \\ (VII) Final approval of manuscript: All authors. \\ "These authors contributed equally to this work. \\ Correspondence to: Ye Gong; Qing Xie. No. 12 Middle Wulumuqi Road, Shanghai 200040, China. Email: drgongye@163.com; neurosurgeon.qing@gmail.com.
}

Background: Resection of meningioma located at primary motor cortex (PMC) is a great challenge to neurosurgeons due to the possibility of motor cortex injury and drainage venous damage during surgery. Here, we presented our experience in resection of PMC meningioma, with the assistance of preoperative multi-model image planning and intraoperative electrophysiological monitor. By these techniques, patients in our study achieved gross tumor resection (GTR) with appropriate motor cortex and drainage venous protection, and the patients recovered well with less neurologic deficits.

Methods: Thirty patients of PMC meningioma were selected for the study during January 2014 to December 2015. All the patients were performed head magnetic resonance imaging (MRI), magnetic resonance venography (MRV) and functional MRI scan before surgery, then all the imaging data were transferred to dextroscope workstation for virtual reality (VR) planning, to display the relationship of tumor, drainage venous and motor cortex. During the operation, somatosensory-evoked potentials (SSEP) was firstly used to locate the central sulcus (CS) and define the PMC area. Then the tumors were carefully removed by avoiding damage the cortex and drainage venous. After tumor resection, motor-evoked potentials (MEP) was applied to evaluate the motor function reservation.

Results: All the patients received preoperative image planning, and the relationships of tumor, drainage venous and motor cortex were clearly presented. With the application of SSEP monitor, the CS was firstly determined before tumor resection. Twenty-five cases in our study achieved radical resection of Simpson I, and 5 cases were Simpson II resection. After tumor resection, MEP monitor was used to confirm the preservation of motor function. Four patients suffered from moderate muscle strength decrease postoperatively, 10 patients experienced slight muscle strength decrease, while other 16 patients shared normal muscle strength as preoperative status. When discharge, those affected patients were recommended for further movement rehabilitation therapy.

Conclusions: Preoperative multi-model image planning and intraoperative electrophysiological monitor are very useful techniques for resection of PMC meningioma. They could help decrease motor cortex damage and protect the drainage venous during surgery, which is much safer and benefit patient for better prognosis.

Keywords: Primary motor cortex (PMC); meningioma; drainage vein; electrophysiological monitor

Submitted Dec 01, 2018. Accepted for publication Dec 11, 2018.

doi: $10.21037 /$ tcr.2018.12.11

View this article at: http://dx.doi.org/10.21037/tcr.2018.12.11 


\section{Introduction}

Meningioma arises from the meninges covering the brain and spinal cord, and ranks the second common primary brain tumors after gliomas, accounting for about $30 \%$ of all brain tumors (1-4). According to the World Health Organization (WHO) classification, most meningiomas are histologically benign (WHO grade I) tumors. However, about $10 \%$ are malignant tumors, for instance, atypical meningioma (WHO grade II) or anaplastic meningioma (WHO grade III) subtypes (5-7). Meningiomas mostly locate at brain convexity, para-sagittal and skull base, which frequently invade surrounding brain tissues and vital neurovascular structures, leading to neurological deficits and requiring surgical intervention. However, those meningiomas located at primary motor cortex (PMC) area are especially challenging because of the possibility of motor cortex damage, drainage venous injury and subsequent neurological deficits after surgery.

Thus, how to preserve the motor cortex and drainage venous for PMC meningioma during operation is a problem. Here, we presented our experiences using preoperative multi-model image planning to clearly display the relationships of tumor, drainage venous and motor cortex, and using intraoperative electrophysiological monitor to protect motor function. Intraoperative electrophysiological monitor is currently widely used in eloquent area glioma surgery, while seldom applied in functional area meningioma resection (8-10). Utilizing somatosensory-evoked potentials (SSEP), central sulcus (CS) could be determined clearly, which divides the PMC and primary sensory cortex. After identifying the CS, the precentral gyrus was considered as PMC, and keeping the PMC intact is very important for preserving the motor function during surgery. Furthermore, we also performed motor-evoked potential (MEP) monitor after tumor resection to confirm the existence of motor function. Combined with these techniques, we could preserve the motor function during surgery.

In our study, we introduced our experience in resection of PMC meningioma with the assistance of preoperative multi-model image planning and intraoperative electrophysiological monitor. Using these techniques, patients in our study achieved gross tumor resection (GTR) with motor cortex and drainage venous preservation, and the patients recovered well with less neurologic complications.

\section{Methods}

\section{Statement of ethics approval}

This study was approved by the Ethics Committee of Huashan Hospital, Fudan University (No. 2012-048), and all the participants gave informed consents.

\section{Patients}

We included a total of 30 patients with meningioma located at PMC region that underwent tumor resection by our team at the Neurosurgery Department of Shanghai Huashan Hospital from January 2014 to December 2015. The base of the meningioma included brain convexity, para-sagittal or falcine, and summary of all the cases were listed in Table 1 . All the cases were pathologically confirmed as meningioma after surgery. All the patients were for initial admission, and didn't receive any preoperative treatment.

\section{Head MRI scanning}

All the patients received head magnetic resonance imaging (MRI) scan after admission, including plain scan and contrast scan. Additionally, head magnetic resonance venography (MRV) was also performed for those patients with meningioma stick to the superior sagittal sinus (SSS). All MRI scans were performed by Siemens clinical 3T MRI scanner (Siemens, Germany). For MRV scan, the three-dimensional time-of-flight spoiled gradient recalled acquisition in the steady-state (3D TOF-SPGR) sequences were utilized with the following scanning parameters: repetition time $(\mathrm{TR})=20 \mathrm{~ms}$, echo time $(\mathrm{TE})=5 \mathrm{~ms}$, field of view $(\mathrm{FOV})=250 \mathrm{~mm}$. Then the images were processed at workstation computers to display the relationships of the tumor, SSS and cortical drainage veins as described in previous literature (11).

Moreover, functional MRI (fMRI) of limb movement was also conducted for the patients. The blood oxygen level dependence (BOLD) echo planning sequence was used and the scanning parameters were as follows: $\mathrm{TR}=3,000 \mathrm{~ms}$, $\mathrm{TE}=30 \mathrm{~ms}, \mathrm{FOV}=200 \mathrm{~mm}$, slice thickness $=3 \mathrm{~mm}$, spaces $=0.8 \mathrm{~mm}$. The movement of thumb and other fingers were provoked to excite the cortical motor area. The images were then processed to display the functional area of hand and foot movement on the cortex, and reveal the influence of motor cortex area by the tumor. 
Table 1 Summary of the 30 patients with primary motor cortex meningioma

\begin{tabular}{lc}
\hline Characteristic & Number \\
\hline Patients & 30 \\
Age, year (range) & $22-72$ \\
Gender (male/female) & $11 / 19$ \\
Symptoms & \\
Weakness/numbness of limbs & 14 \\
Headache/dizziness & 10 \\
Seizure attack & 6 \\
Tumor location & 5 \\
Para-sagittal & 6 \\
Para-falx & 1 \\
Para-sagittal/falx & 18 \\
Convexity & 16 \\
Extent of resection & \\
Simpson I & \\
Simpson II & 25 \\
Preoperative muscle strength & 5 \\
Normal & \\
Weakness & 10 \\
Postoperative muscle strength & \\
Moderate decrease & \\
\hline Slight decrease & \\
\hline
\end{tabular}

\section{Virtual reality (VR) planning}

After MRI scan, all the imaging data (MRI, MRV and fMRI) were collected and uploaded to the VR workstation (Dextroscope, Volume Interactions, Singapore) (12). The images were then processed within the RadioDexter software in order to display the stereoscopic images of tumor, its adjacent vein and functional cortex. Surgical planning could be also performed within this system to simulate the whole process of tumor resection. With such simulation, the neurosurgeon could mentally have confidence in resection of tumor while protecting those critical structures before operation. This system had proved immensely assistance in surgical planning for brain tumors.

\section{Intraoperative neuronavigation}

The StealthStation Treon Neuronavigator (Medtronic Navigation, Inc.) was used for all surgeries (12). The day before operation, at least 6 reference fiducials were placed on the patient's scalp, and navigation MRI scan was conducted for all patients, including MRI, MRV and fMRI scan sequences, which were introduced above. Then all the images data were input into the navigation system. Registration was firstly performed in the operating room by touching the markers with the probe while indicating to the surgical workstation where marker was being touched. After registration, the meningioma on the scalp projection was localized for surgical incision. Within the workstation, the tumor and its adjacent drainage vein or functional motor cortex could be observed from different points of view for intraoperative navigation in real-time.

\section{Electrophysiological monitor and surgical manipulation}

A current generator (Eclipse, Medtronic, Inc.) was utilized for intraoperative electrophysiological monitor during operation. After induction of anesthesia, transcortical stimulation probes were placed according to the protocol. Upon opening the dura, we firstly used SSEP monitor to locate the CS, and determined the PMC area. To identify the CS, we used constant-current/single-pulse stimulation with the following parameters: intensity $=20 \mathrm{~mA}$; duration $=300 \mu$ s; frequency $=4.7 \mathrm{~Hz}(11)$.

Then, tumor was debulked and resected under neuronavigation guidance, taking great care into avoiding eloquent cortex damage and protecting the drainage veins. We applied the CUSA Excel ultrasonic aspiration system (Integra Lifesciences Corporation) for hard tumor resection, which could decrease damage to the cortex and vessels (13). Simpson I resection was achieved for most patients with $\mathrm{PMC}$ meningioma and drainage veins were preserved. Although several meningiomas had invaded the SSS, Simpson grade I resection was still the primary surgical goal for such patients.

After tumor resection, MEP monitor was performed to verify the functional ability of hand and foot movement. Direct cortical stimulation was introduced. The stimulation was initiated by constant-current. The stimulus duration was $75 \mu \mathrm{s}$. The stimulating frequency was $1 \mathrm{~Hz}$ by hand-control. The motor response was evaluated by electromyographic recording. 


\section{Follow up}

All the patients received postoperative therapy and nursing cares, and achieved satisfactory recovery. They were then evaluated for two years' follow-up. Patients who had persistent decrease in muscle strength postoperatively were referred to rehabilitation.

\section{Results}

\section{Clinical outcomes}

All the 30 patients' clinical data were listed in Table 1, including 19 female patients and 11 male patients. The age arranged from 22 to 72 years old, with the average age of 51 years old. At admission, 14 patients complained symptoms of muscle strength decrease (Table 1), while another 16 patients presented with headache, dizziness or seizure attack.

After operation, no muscle strength impairment was observed among the 16 patients $(53.33 \%)$ without muscle strength impairment preoperatively. However, in another 14 patients, 4 patients $(13.33 \%)$ suffered from moderate muscle strength decrease, and 10 patients $(33.33 \%)$ showed slight muscle strength decrease. All the 14 affected patients $(46.67 \%)$ were recommended for physical therapy and rehabilitation treatment after discharge. Till the last follow-up, among the 14 affected patients, 13 patients achieved well recovery of motor function without paralysis except 1 patient $(3.33 \%)$.

\section{Preoperative imaging preparation}

All the patients received preoperative head MRI scan, including plain and contrast MRI scan, MRV scan, fMRI scan, and neuronavigation MRI scan. In our study, 5 cases were para-sagittal meningiomas, 6 cases were para-falx meningiomas, and 1 case invaded both the SSS and falcine. Another 18 cases were convexity meningiomas (Table 1).

Here, we selected case No. 2 as an example to explain how to prepare preoperative MRI for such patients. Case No. 2 patient was a 52-year-old female suffered from gradual weakness of her left limbs for about one year. After admission, she underwent plain and contrast MRI scan (Figure 1A,B) and right-side PMC meningioma was suspected. Brain MRV was also performed for her because the tumor was adhered to the SSS (Figure 1C), showing that the SSS was compressed by the tumor and narrowed.
Protecting SSS and other vital drainage veins are very important. Moreover, fMRI scan revealed PMC area was pushed way posterior by the tumor.

All the imaging data were then transferred to VR workstation in order to display the stereotactic images of the tumor, its adjacent venous structures and functional brain regions (Figure 1D). The day before operation, neuronavigation MRI scan was conducted for intraoperative reference. From neuronavigation MRI images, the tumor, and its relationship with PMC area and SSS were clearly displayed (Figure 1E,F).

In some cases, we used neuronavigation MRV sequence to furtherly display the relationship of the tumor, SSS and drainage veins (Figure 2A,B). Combined with all these above preoperative MRI images, we could plan the whole surgical process, in order to achieve maximum tumor resection while minimum motor cortex and drainage veins damage.

\section{Intraoperative electrophysiological monitor}

During the operation, we firstly utilized SSEP monitor to identify the CS before opening the dura (Figure $3 A, B$ ), and verified the $\mathrm{PMC}$ area combined with intraoperative MRI navigation (Figure $1 E$ and $1 F$ ). Then tumor resection was carried out, with great care taken into avoid damaging adjacent functional cortex and drainage venous during operation.

Simpson I resection was the prerequisite aim for the 18 cases of convexity meningiomas and 6 cases of parafalx meningiomas. For the other 6 cases invaded the SSS, Simpson I resection was still the primary surgical goal. As to the No. 2 case, we resected the tumor from the nonfunctional area, intra-tumor debulk strategy was firstly performed, we next separated the tumor from adjacent cortex along its boundary and carefully separated the drainage veins from the tumor, then the tumor was removed by pieces with repeated procedures. After tumor resection, we dealt with the tumor adhesion to the SSS, resect the tumor invaded into the SSS and finally repair the SSS wall with autologous fascia (Figure $4 A, B, C, D$ ). Simpson I resection was achieved for this case, while motor cortex and vital drainage veins were protected well. In our report, 25 cases achieved radical resection of Simpson I, and 5 cases were Simpson II resection.

Then, we performed MEP monitor to verify the preservation of motor function of hand and foot (Figure $5 A, B$ ) after tumor resection. 

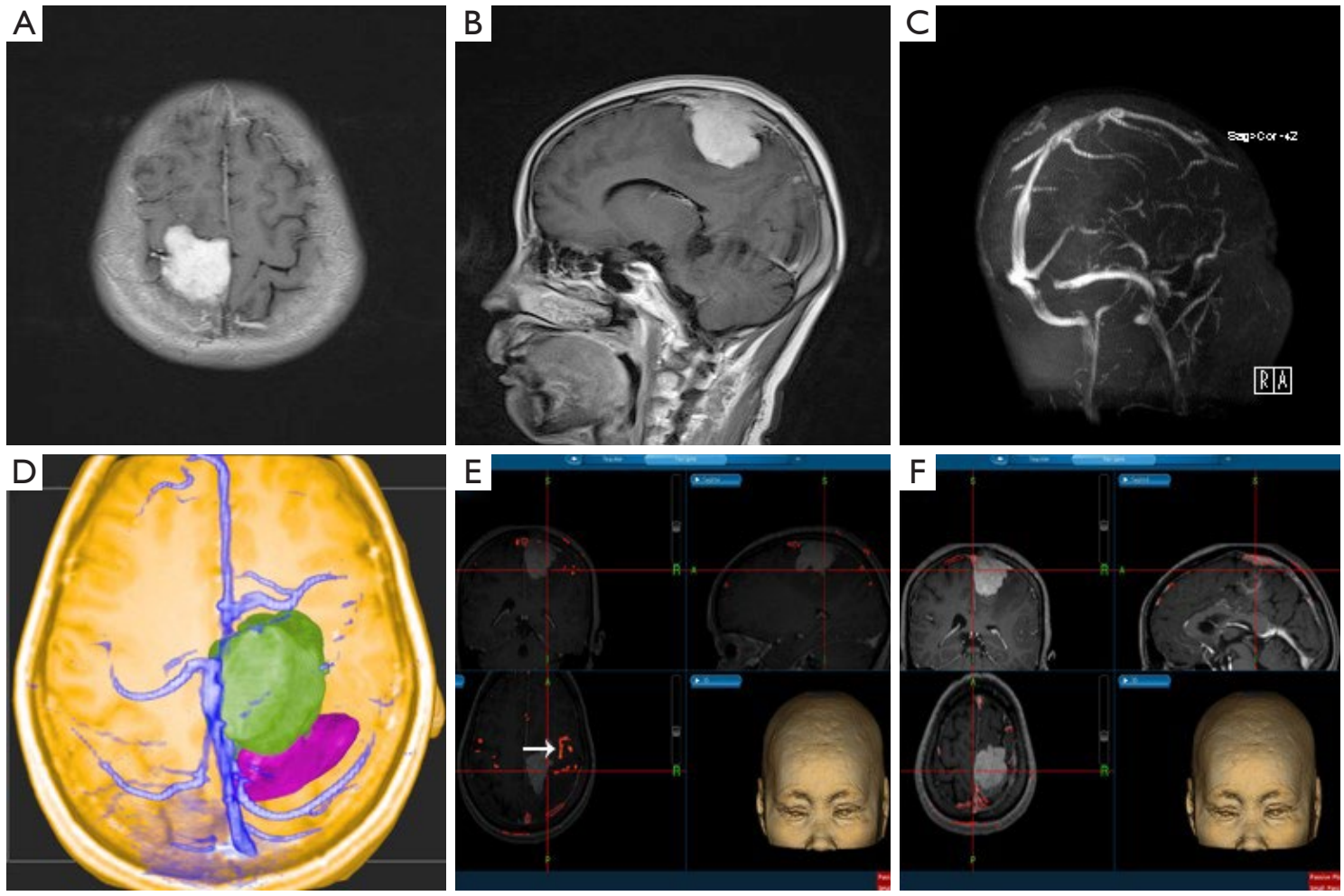

Figure 1 Preoperative imaging preparation for primary motor cortex (PMC) meningioma. (A,B) Head contrast MRI scan showed right frontal, para-sagittal meningioma. (C) Head MRV scan showed the superior sagittal sinus was invaded by the tumor and narrowed. (D) Virtual reality image displayed the clear relationship of tumor (green), motor function cortex (purple) and superior sagittal sinus (blue). (E) Neuronavigation displayed the motor function cortex (as the white arrow indicated) was pushed away by the tumor. (F) Neuronavigation showed the superior sagittal sinus was affected by the tumor. MRI, magnetic resonance imaging; MRV, magnetic resonance venography.
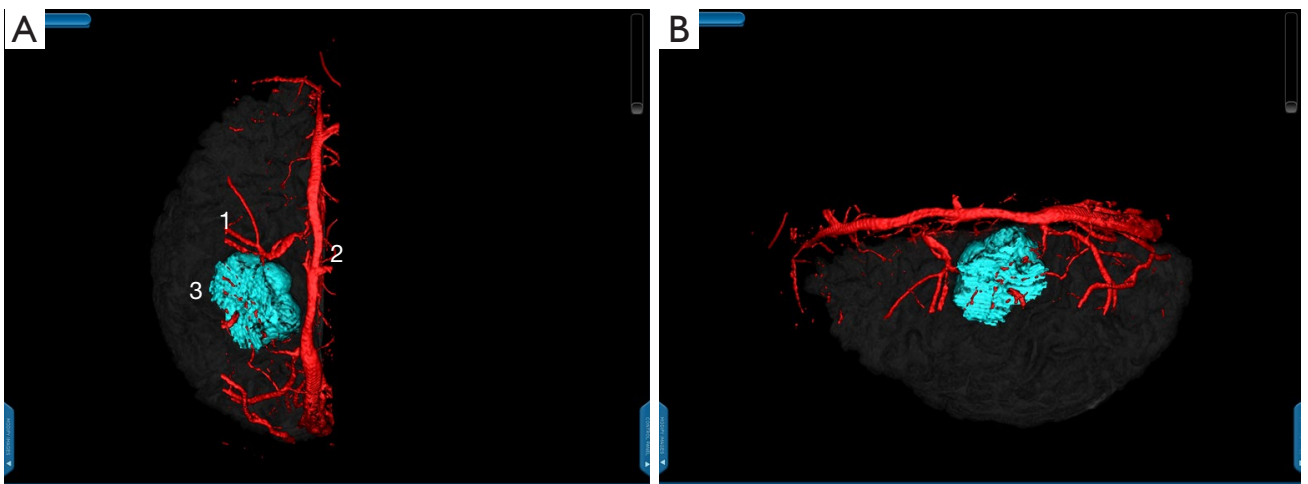

Figure 2 Neuronavigation MRV scan for primary motor cortex meningioma. (A) Axial view of left primary motor cortex meningioma, the relationship of tumor [3], drainage vein [1] and superior sagittal sinus [2] was clearly displayed; (B) sagittal view of the above relationships. 

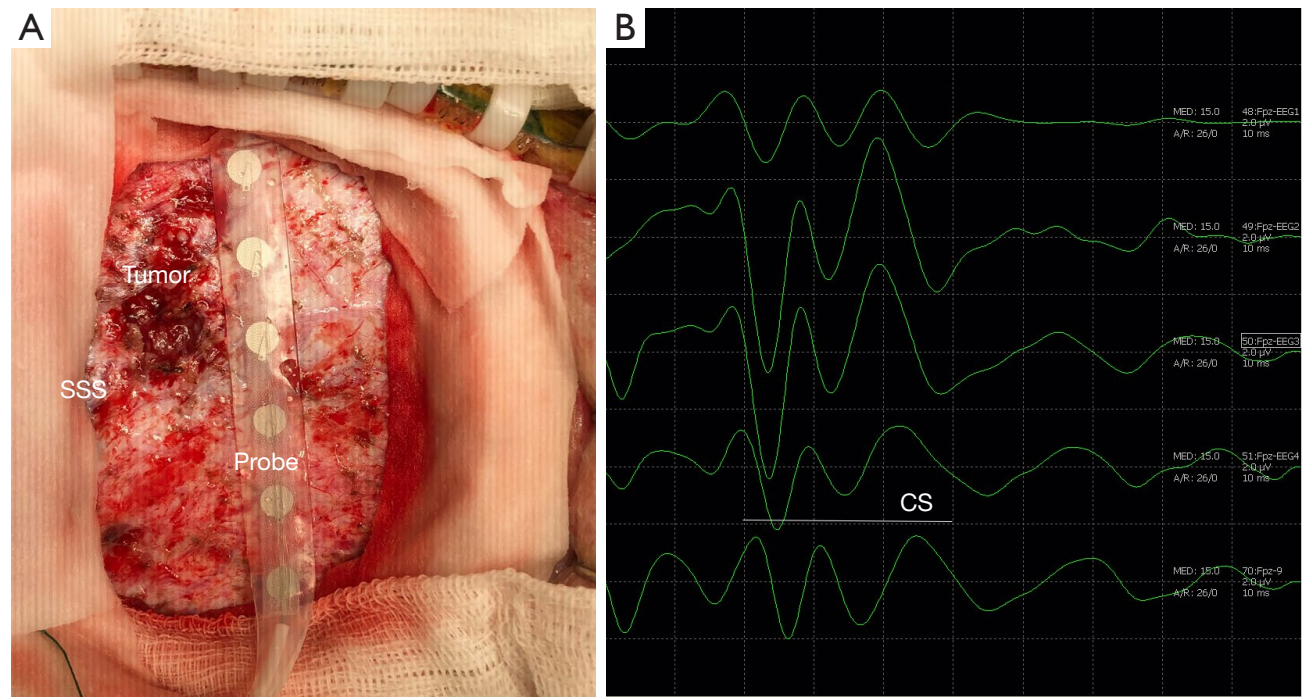

Figure 3 Somatosensory evoked potentials (SSEP) monitor before resection of PMC meningioma. (A) Upon dura opening, somatosensory evoked potentials (SSEP) monitor was used to determine the central sulcus (CS) and the precentral gyrus, and the tumor could be carefully removed by avoiding these important structures. (B) According to the electrophysiological monitor results, the CS could be easily determined (as the white line showed). PMC, primary motor cortex.
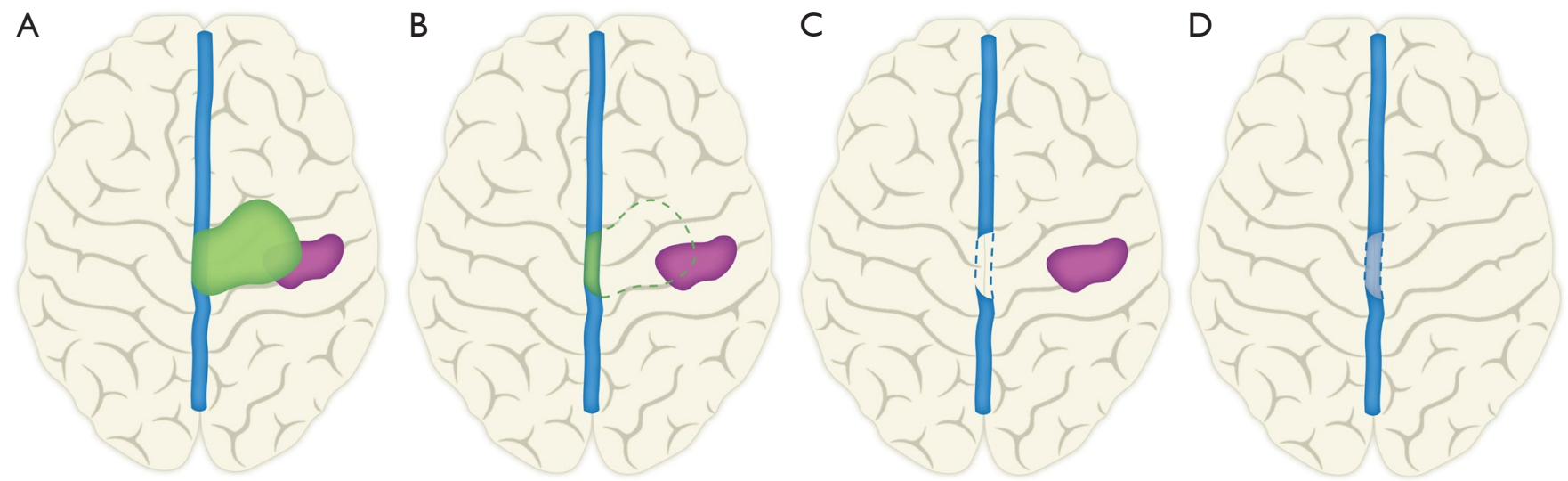

Figure 4 Sketch map for resection of PMC meningioma invading the SSS. (A) Sketch picture showed PMC meningioma (green) invaded superior sagittal sinus (SSS, blue), and pushed away the functional cortex (purple). (B) The tumor was resected along the non-functional area while protecting the drainage vein and functional cortex. (C) Then the tumor part invading the SSS was removed by temporally occlude the SSS with gelatin sponge. (D) Finally, the SSS was repaired by autologous fascia tissue. PMC, primary motor cortex.

\section{Discussion}

Brain tumors located at PMC area, including meningioma, can involve both the motor cortex and drainage veins, and often lead to neurological deficits. Therefore, surgical resection of meningioma in such eloquent areas remains a great challenge to neurosurgeons. Many advanced techniques, including multiple preoperative venous image planning and intraoperative neuronavigation, have allowed identification of vital functional cortex and vital venous structures, in order to maximize tumor resection and minimize vital structures injury. Intraoperative electrophysiological monitor and brain mapping have gained popularity in recent years for brain tumor resection, such as gliomas that invaded motor cortex and its subcortical fiber tracts (14-16). However, its application in resection of PMC meningioma was seldom 

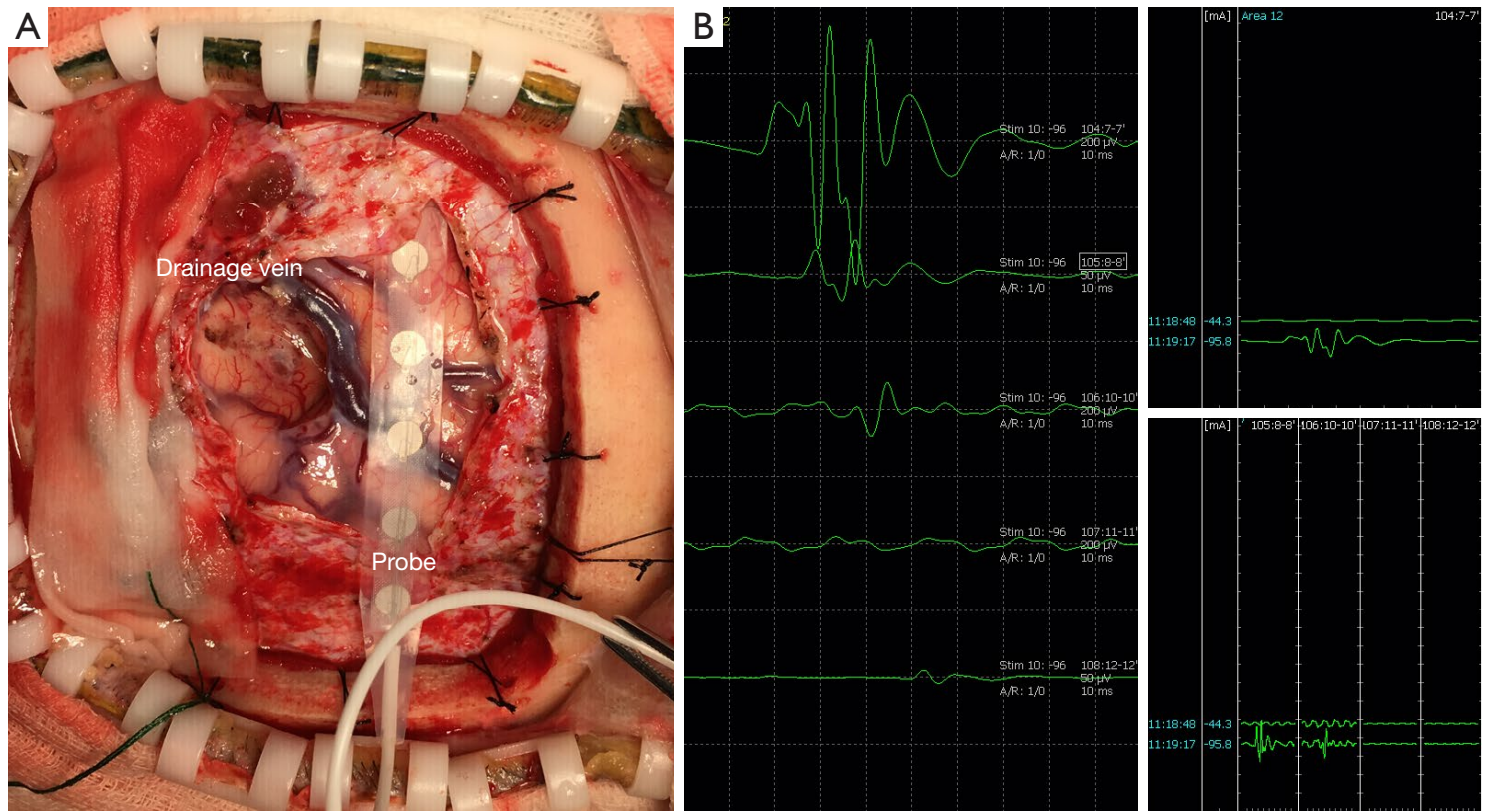

Figure 5 Motor-evoked potentials (MEP) monitor for verification of motor function after operation. (A) After tumor resection, MEP monitor was performed to verify the preservation of motor function. (B) As the electrophysiological monitor results showed, there were currents of hand and foot, indicating the preservation of motor function.

reported (17). PMC meningioma is more difficult because of its complex anatomy of tumor and its surrounding structures, for instance, motor cortex, SSS and several drainage veins. Protection of these structures during tumor resection is the optimal task, and is the key for motor function preservation.

In our study, we included 30 patients with PMC meningioma. The base of the meningioma contained brain convexity, para-sagittal and falcine. Prior to the operation, all patients received head MRI scan, including plain and contrast MRI scan, MRV scan and fMRI scan, and neuronavigation MRI scan. All the imaging data were then transferred to VR workstation to display the stereoscopic images of the tumor, its adjacent functional brain cortex and venous structures (Figures 1 and 2). We hoped to demonstrate that such multi-model preoperative imaging planning would benefit the neurosurgeons during surgery. It would aid the neurosurgeon in identifying the tumor base, the distribution of the venous structures, and the location of the motor cortex region.

During the operation, we firstly utilized SSEP monitor to map the CS before dura opening (Figure 3), and to define the functional brain cortex. Prior to the implementation of intraoperative electrophysiological monitor in meningioma surgery, it was often difficult to define the CS and PMC area intraoperatively due to brain shift by tumor compression. With the application of electrophysiological monitor, the functional brain cortex could be easily and reliably determined during operation prior to tumor resection. We could then carefully separate the tumor from non-functional cortex along its border, keep the drainage veins intact, and remove the tumor by pieces till Simpson I/II resection. For those cases invaded the SSS, Simpson I resection was still the primary surgical goal according to the surgical plan (Figure 4). Consequently, the functional brain cortex and the drainage venous structures could be well preserved during tumor resection.

After tumor resection, we performed MEP monitor to verify the functional preservation of limb movement (Figure 5). In our study, all the cases showed no significant change in MEP currents after tumor resection, indicating that the PMC area were protected during operation. Four patients exhibited moderate muscle strength decrease after surgery, which was caused by severe brain edema. However, they got improvement after 12 months of physical and rehabilitation therapy except one patient. There was one large drainage vein damage during operation in this patient. This patient was still in physical exercise by our last follow- 
up. Ten patients suffered from slight muscle strength decrease, which was caused by transient brain edema after surgery. And they were also recovered 3-6 months after discharge. The other 16 patients shared the same muscle strength as preoperative status. After two years' follow-up, all the patients recovered well without tumor recurrence and neurological deficits except the above one patient.

\section{Conclusions}

In conclusion, combinations of multiple preoperative venous image planning and intraoperative electrophysiological monitor are very critical for resection of PMC meningiomas (17-20). Preoperative venous image planning could clearly display the relationship of tumor, SSS and drainage veins before surgery. While using intraoperative electrophysiological monitor, the CS and PMC could be determined, protected and confirmed during tumor resection. These techniques could help decrease motor cortex damage and protect the drainage veins, which would benefit patient for long-term period of prognosis with less neurological deficits.

\section{Acknowledgments}

We are grateful to Dr. Geng Xu for his assistance in intraoperative electrophysiological monitor and data analysis.

Funding: This work was also supported by grants (No. $81772674,81372707)$ from National Nature Science Foundation, and grants (No. 15140902200, 16140903000) from Shanghai Committee of Science and Technology.

\section{Footnote}

Conflicts of Interest: All authors have completed the ICMJE uniform disclosure form (available at http://dx.doi. org/10.21037/tcr.2018.12.11). The authors have no conflicts of interest to declare.

Ethical Statement: The authors are accountable for all aspects of the work in ensuring that questions related to the accuracy or integrity of any part of the work are appropriately investigated and resolved. The study was conducted in accordance with the Declaration of Helsinki (as revised in 2013). This study was approved by the Ethics Committee of Huashan Hospital, Fudan University (No. 2012-048), and all the participants gave informed consents.

Open Access Statement: This is an Open Access article distributed in accordance with the Creative Commons Attribution-NonCommercial-NoDerivs 4.0 International License (CC BY-NC-ND 4.0), which permits the noncommercial replication and distribution of the article with the strict proviso that no changes or edits are made and the original work is properly cited (including links to both the formal publication through the relevant DOI and the license). See: https://creativecommons.org/licenses/by-nc-nd/4.0/.

\section{References}

1. Wang DJ, Xie Q, Gong Y, et al. Histopathological classification and location of consecutively operated meningiomas at a single institution in China from 2001 to 2010. Chin Med J (Engl) 2013;126:488-93.

2. Zhu H, Xie Q, Zhou Y, et al. Analysis of prognostic factors and treatment of anaplastic meningioma in China. J Clin Neurosci 2015;22:690-5.

3. Tang H, Sun H, Xie L, et al. Intraoperative ultrasound assistance in resection of intracranial meningiomas. Chin J Cancer Res 2013;25:339-45.

4. Tang H, Zhu H, Wang X, et al. KLF4 is a tumor suppressor in anaplastic meningioma stem-like cells and human meningiomas. J Mol Cell Biol 2017;9:315-24.

5. Louis DN, Perry A, Reifenberger G, et al. The 2016 World Health Organization Classification of Tumors of the Central Nervous System: a summary. Acta Neuropathol 2016;131:803-20.

6. Riemenschneider MJ, Perry A, Reifenberger G. Histological classification and molecular genetics of meningiomas. Lancet Neurol 2006;5:1045-54.

7. Mawrin C, Perry A. Pathological classification and molecular genetics of meningiomas. J Neurooncol 2010;99:379-91.

8. Chacko AG, Thomas SG, Babu KS, et al. Awake craniotomy and electrophysiological mapping for eloquent area tumours. Clin Neurol Neurosurg 2013;115:329-34.

9. Maesawa S, Fujii M, Nakahara N, et al. Intraoperative tractography and motor evoked potential (MEP) monitoring in surgery for gliomas around the corticospinal tract. World Neurosurg 2010;74:153-61.

10. Hayashi Y, Nakada M, Kinoshita M, et al. Functional Reorganization in the Patient with Progressing Glioma of the Pure Primary Motor Cortex: A Case Report with Special Reference to the Topographic Central Sulcus Defined by Somatosensory-Evoked Potential. World Neurosurg 2014;82:536.e1-4.

11. Zhu FP, Wu JS, Song YY, et al. Clinical application of 
motor pathway mapping using diffusion tensor imaging tractography and intraoperative direct subcortical stimulation in cerebral glioma surgery: a prospective cohort study. Neurosurgery 2012;71:1170-83.

12. Tang HL, Sun HP, Gong Y, et al. Preoperative surgical planning for intracranial meningioma resection by virtual reality. Chin Med J (Engl) 2012;125:2057-61.

13. Tang H, Zhang H, Xie Q, et al. Application of CUSA Excel ultrasonic aspiration system in resection of skull base meningiomas. Chin J Cancer Res 2014;26:653-7.

14. Wu JS, Zhou LF, Tang WJ, et al. Clinical evaluation and follow-up outcome of diffusion tensor imaging-based functional neuronavigation: a prospective, controlled study in patients with gliomas involving pyramidal tracts. Neurosurgery 2007;61:935-48.

15. Prabhu SS, Gasco J, Tummala S, et al. Intraoperative magnetic resonance imaging-guided tractography with integrated monopolar subcortical functional mapping for resection of brain tumors. Clinical article. J Neurosurg 2011;114:719-26.

16. Nossek E, Korn A, Shahar T, et al. Intraoperative

Cite this article as: Tang H, Xu F, Lin L, Zhu H, Hua L, Luan S, Xie Q, Gong Y. Intra-operative motor function preservation for resection of primary motor cortex meningioma. Transl Cancer Res 2018;7(6):1666-1674. doi: 10.21037/tcr.2018.12.11 mapping and monitoring of the corticospinal tracts with neurophysiological assessment and 3-dimensional ultrasonography-based navigation. Clinical article. J Neurosurg 2011;114:738-46.

17. Ostrý S, Netuka D, Benes V. Rolandic area meningioma resection controlled and guided by intraoperative cortical mapping. Acta Neurochir (Wien) 2012;154:843-53.

18. Gazzeri R, Galarza M, Fiore C, et al. Use of tissueglue-coated collagen sponge (TachoSil) to repair minor cerebral dural venous sinus lacerations: technical note. Neurosurgery 2015;11 Suppl 2:32-6.

19. Türe H, Harput MV, Bekiroğlu N, et al. Effect of the degree of head elevation on the incidence and severity of venous air embolism in cranial neurosurgical procedures with patients in the semisitting position. J Neurosurg 2018;128:1560-9.

20. Debernardi A, Quilici L, La Camera A, et al. Torcular Meningioma with Multi-Venous Sinus Invasion: Compensatory Drainage Veins and Surgical Strategy. World Neurosurg 2018;109:451-4. 